\title{
High-Resolution Nanoscale Solid-State Nuclear Magnetic Resonance Spectroscopy
}

\author{
William Rose, ${ }^{1}$ Holger Haas, ${ }^{2,3}$ Angela Q. Chen, ${ }^{1}$ Nari Jeon, ${ }^{4}$ Lincoln J. Lauhon, ${ }^{4}$ \\ David G. Cory, ${ }^{3,5,6,7}$ and Raffi Budakian ${ }^{1,2,3,6,7, *}$ \\ ${ }^{1}$ Department of Physics, University of Illinois at Urbana-Champaign, Urbana, Illinois 61801-3080, USA \\ ${ }^{2}$ Department of Physics, University of Waterloo, Waterloo, Ontario, Canada, N2L3G1 \\ ${ }^{3}$ Institute for Quantum Computing, University of Waterloo, Waterloo, Ontario, Canada, N2L3G1 \\ ${ }^{4}$ Department of Materials Science and Engineering, Northwestern University, \\ Evanston, Illinois 60208, USA \\ ${ }^{5}$ Department of Chemistry, University of Waterloo, Waterloo, Ontario, Canada, N2L3G1 \\ ${ }^{6}$ Perimeter Institute for Theoretical Physics, Waterloo, Ontario, Canada, N2L2Y5 \\ ${ }^{7}$ Canadian Institute for Advanced Research, Toronto, Ontario, Canada, M5G1Z8
}

(Received 20 July 2017; revised manuscript received 10 January 2018; published 26 February 2018)

\begin{abstract}
We present a new method for high-resolution nanoscale magnetic resonance imaging (nano-MRI) that combines the high spin sensitivity of nanowire-based magnetic resonance detection with high-spectralresolution nuclear magnetic resonance (NMR) spectroscopy. Using a new method that incorporates average Hamiltonian theory into optimal control pulse engineering, we demonstrate NMR pulses that achieve high-fidelity quantum control of nuclear spins in nanometer-scale ensembles. We apply this capability to perform dynamical decoupling experiments that achieve a factor of 500 reduction of the proton-spin resonance linewidth in a $(50-\mathrm{nm})^{3}$ volume of polystyrene. We make use of the enhanced spin coherence times to perform Fourier-transform imaging of proton spins with a one-dimensional slice thickness below $2 \mathrm{~nm}$.
\end{abstract}

DOI: 10.1103/PhysRevX.8.011030

Subject Areas: Magnetism, Nanophysics

\section{INTRODUCTION}

Magnetic resonance imaging (MRI) is a powerful noninvasive technique that has transformed our ability to study the structure and function of biological systems. Key to its success has been the unique ability to combine imaging with magnetic resonance spectroscopy. This capability has led to a host of different imaging modalities that rely on the ability to distinguish the interaction of spins with their local chemical environment. Although it remains a significant challenge, there is considerable interest in extending the capabilities of MRI spectroscopy to the nanometer scale because it would provide a fundamentally new route for determining the structure and function of complex biomolecules.

A key challenge for nano-MRI is to have the ability to sense nanometer-scale ensembles of nuclear spins. In recent years, a number of different approaches to ultrasensitive spin detection have been developed, including magnetic resonance force microscopy (MRFM) [1-3] and nitrogen-vacancy magnetometry [4-6]. These techniques

\footnotetext{
*rbudakian@uwaterloo.ca
}

Published by the American Physical Society under the terms of the Creative Commons Attribution 4.0 International license. Further distribution of this work must maintain attribution to the author(s) and the published article's title, journal citation, and DOI. have improved upon the spin-detection sensitivity of conventional inductively detected magnetic resonance by more than 8 orders of magnitude, culminating in the detection of single electron [1] and proton [7] spins, and MRI of tobacco mosaic virus particles with sub-10-nm spatial resolution [2]. As demonstrated by the virus MRI result, MRFM holds promise for studying biological samples in part because of its capability to image sufficiently long length scales, up to around $100 \mathrm{~nm}$, with very fine resolution. To fully realize the potential of nano-MRI, however, we need a platform that combines ultrasensitive spin detection with high-resolution magnetic resonance spectroscopy.

As the spin detection and imaging success of MRFM were being established, there were also efforts to demonstrate its utility for dipolar [8,9], quadrupolar [10], and chemical-shift [11] spectroscopy by employing magic-echo (ME) pulse sequences and other techniques developed for conventional solid-state magnetic resonance imaging. These experiments were able to achieve spectral resolution under $1 \mathrm{kHz}$, showing the spectroscopy possibilities of MRFM, but were limited to relatively large spin ensembles with spatial resolution around $1 \mu \mathrm{m}$. As Eberhardt et al. write, the static gradient sources used in many MRFM experiments complicate spectroscopy attempts by interfering with the pulses needed to perform dynamical decoupling pulse sequences [11]. They solved this problem by moving the gradient 
source out of range of their sample during the encoding pulse sequence using a piezoelectric actuator.

We avoid issues arising from static gradient sources by generating magnetic field gradients using a currentfocusing field gradient source (CFFGS), which generates large magnetic fields and gradients on nanometer length scales by focusing electric currents through a narrow $(\sim 100 \mathrm{~nm})$ metallic constriction. CFFGS devices can generate very large time-dependent magnetic fields $(\sim 0.1 \mathrm{~T})$ and magnetic field gradients $\left(\sim 10^{6} \mathrm{~T} / \mathrm{m}\right)$ over a wide frequency bandwidth (DC-GHz) [12] in nanometer-scale volumes. The gradient strengths generated are comparable to those from the powerful static gradient nanomagnets used in other MRFM experiments [2]. As Nichol et al. demonstrated in a previous two-dimensional nano-MRI experiment, the strong magnetic fields produced by a CFFGS allow for rapid spin manipulations, while the large field gradients enable very sensitive spin detection on spin ensembles as large as $(50 \mathrm{~nm})^{3}$ [3].

Most conventional MRI techniques, as well as the nanoMRI measurements by Nichol et al., employ Fouriertransform imaging to construct the spin density. In Fourier-transform MRI, the minimum feature size that can be resolved is inversely proportional to the product of the magnetic field gradient used for phase encoding and the available encoding time. Hence, to achieve high spatial resolution, one needs the ability to generate large encoding gradients and maintain long spin coherence times. Achieving high spatial resolution in solid-state samples is particularly challenging because the strong dipole-dipole interactions give rise to short coherence times. In the nano-MRI experiment by Nichol et al., the encoding time was limited by the spin dephasing time of $T_{2}^{*}=14 \mu \mathrm{s}$, dominated by the strong homonuclear dipolar interaction between proton spins in polystyrene, which limited the spatial resolution to about $10 \mathrm{~nm}$ [3].

To image with finer spatial resolution and to enable highresolution spectroscopy, dipolar interactions between spins must be decoupled. The solid-state MRI community has developed a variety of decoupling techniques. In particular, the ME pulse sequence has been used to decouple both homonuclear and heteronuclear interactions. In addition, the ME sequence has long laboratory and rotating-frame spin evolution periods, during which gradient pulses may be applied for spatial encoding. Dynamical decoupling sequences such as the ME typically require implementing a set of unitary operations on the spin ensemble. The most common way for implementing a unitary is by applying a constant amplitude resonant radio frequency (rf) excitation, also known as a hard pulse. For a hard pulse to implement the same unitary for each spin within the ensemble, the amplitude of the rf field $\left(B_{1}\right)$ must be uniform throughout the sample. In our nano-MRI platform, we rely on the CFFGS to produce the rf excitation. While the CFFGS is capable of producing intense magnetic fields, the large $B_{1}$ variation over the sample volume makes the use of hard pulses unfeasible.

Many experimental limitations, including $B_{1}$ variation, can be efficiently dealt with using pulses designed with optimal control theory (OCT). Provided one has sufficient knowledge of the experimental conditions-such as resonance offsets, bandwidth limitations, and $B_{1}$ dispersionOCT pulses can be designed to perform rapid, robust unitary transformations.

In this paper, we present a new method for high-fidelity quantum control of nanoscale ensembles of nuclear spins using OCT-engineered pulses that utilize the strong magnetic fields produced by the CFFGS. We employ a new extension of OCT that incorporates average Hamiltonian theory (AHT) to allow for control of the average spin Hamiltonian over the duration of the pulse. We use this capability to perform dynamical decoupling during OCT pulses that have duration comparable to $T_{2}^{*}$. By incorporating OCT-AHT $\pi / 2$ pulses into magic-echo sequences, we narrow the proton-resonance linewidth by a factor of 500 , from $29 \mathrm{kHz}$ to $57 \mathrm{~Hz}$, in a $(50-\mathrm{nm})^{3}$ volume of polystyrene. We make use of the enhanced spin coherence times to perform Fourier imaging of proton spins in polystyrene with a one-dimensional slice thickness between $1.8 \mathrm{~nm}$ and $2.6 \mathrm{~nm}$ within a $35-\mathrm{nm}$ region of the sample. This work serves as a demonstration of the power of OCT-AHT to achieve high-fidelity spin control for spectroscopy and imaging in CFFGS-based nano-MRI systems.

\section{APPARATUS}

As shown in Fig. 1(a), we used an apparatus very similar to that described in our previous nano-MRI work [3]. Force-detected magnetic resonance measurements were performed using silicon nanowire (SiNW) mechanical resonators, grown via the vapor-liquid-solid method. The characteristics of the SiNW resonators are discussed in Appendix A. For the NMR measurements, the tip of the SiNW was coated with a thin layer of polystyrene dissolved in diethyl phthalate.

To generate time-varying magnetic fields and magnetic field gradients, we fabricated a CFFGS device by electronbeam lithography and liftoff of $5-\mathrm{nm} / 80-\mathrm{nm}$ thick Ti/Ag films deposited on a $\mathrm{MgO}(100)$ substrate by electron-beam evaporation. The device contained a 150-nm-wide and 100nm-long constriction, which served to focus electrical currents to produce the magnetic fields used for spin detection and control. A scanning electron micrograph (SEM) of the CFFGS is shown in Fig. 1(b).

During the measurement period, a 70-mA peak current was applied to the CFFGS at the resonance frequency of the $\mathrm{SiNW}$, which produced a time-dependent field gradient having a peak amplitude of $d B_{z} / d x \simeq 1.0 \times 10^{6} \mathrm{~T} / \mathrm{m}$ near the tip of the SiNW located $55 \mathrm{~nm}$ from the surface of the CFFGS. In Sec. III of Ref. [13], we present a detailed discussion of the fields produced by the CFFGS. 

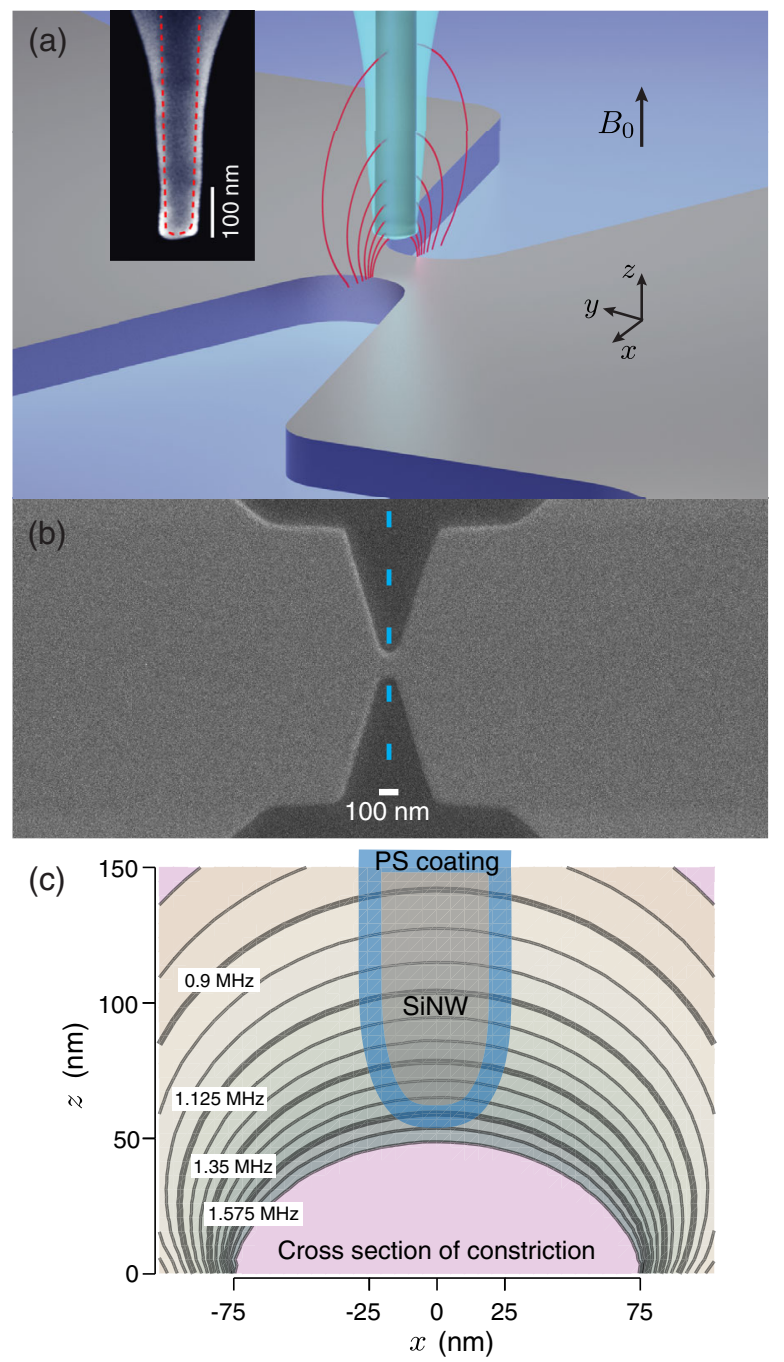

FIG. 1. (a) Schematic rendering of the experimental apparatus, showing the polystyrene-coated nanowire, the CFFGS, and the static field $B_{0}$ used for the magnetic resonance experiments. The lines near the constriction, shown in red, represent contours of constant Rabi frequency $\left[\omega_{1} /(2 \pi)\right]$ generated by flowing rf current through the CFFGS at the Larmor frequency. (Inset) SEM of a representative SiNW tip coated with polystyrene. The outline of the SiNW is shown by the dashed line. (b) SEM of the CFFGS. (c) Schematic of polystyrene-coated nanowire and calculated proton Rabi frequency as a function of $x$ and $z$ positions relative to the center of the constriction [dashed line in (b)]. The Rabi frequencies are given by $\omega_{1}(\vec{r}) /(2 \pi)=\gamma_{p} B_{1}(\vec{r}) /(2 \pi)$, where $B_{1}(\vec{r})=\sqrt{B_{x}^{2}(\vec{r})+B_{y}^{2}(\vec{r})} / 2$ is the amplitude of the circularly polarized rf field. Field values were calculated for 50-mA-pk rf current flowing through the CFFGS.

Magnetic resonance measurements were made using a static external field of $B_{0}=1.13 \mathrm{~T}$, corresponding to a proton Larmor frequency of $48 \mathrm{MHz}$. The rf magnetic field used for spin control was produced by applying 50-mA peak current to the CFFGS, corresponding to
$B_{1}=37 \mathrm{mT}$ and a Rabi frequency of $\omega_{1} /(2 \pi)=$ $\gamma_{p} B_{1} /(2 \pi)=1.58 \mathrm{MHz}$ for proton spins near the tip of the SiNW, where $\gamma_{p}=2 \pi \times 42.6 \mathrm{MHz} / \mathrm{T}$ is the proton gyromagnetic ratio.

Figure 1(c) shows a cross section of the proton Rabi frequency distribution at the center of the CFFGS. The magnetic fields produced by the CFFGS are calculated using the COMSOL Multiphysics finite-element simulation software. The simulations are based on the dimensions of the CFFGS as shown in the SEM image in Fig. 1(b). To confirm the validity of the simulated Rabi field distribution, we performed a one-dimensional imaging experiment of the spins near the tip of the SiNW. The details of this measurement are presented in Sec. VI.

The measurements took place in high vacuum at a base temperature of $4.2 \mathrm{~K}$. We observed approximately $1-2 \mathrm{~K}$ heating of the SiNW because of the laser. The polystyrenecoated SiNW was positioned approximately $50 \mathrm{~nm}$ above the center of the CFFGS using piezoelectric nanopositioners and scanners.

The OCT pulses designed for these measurements occupy a wide bandwidth $(\sim 10 \mathrm{MHz})$. We found that waveform distortions caused by standing waves in the cables between the room-temperature electronics and the CFFGS device can severely degrade the performance of the OCT pulses. We therefore developed a force-detection technique that allowed us to characterize the complex transfer function of the $\mathrm{rf}$ current flowing through the CFFGS. Although the details of the technique will be presented in a separate publication, a brief description is provided in Sec. VI of Ref. [13]. In Sec. II of Ref. [13], we describe the electronics used for generating the OCT and the spin-detection waveforms.

\section{SPIN MEASUREMENT}

In this work, we measure the polarization that results from the statistical imbalance of the spin projection in the direction parallel to the static external field $B_{0}$ ( $z$ direction). The advantage of detecting the statistical polarization is twofold. In an ensemble of $N S=1 / 2$ spins, the average statistical imbalance between the two spin projections $m_{s}= \pm 1 / 2$ is $\sqrt{N}$. In small ensembles, the average statistical polarization can be larger than the thermal polarization. For the smallest ensembles measured in this work, corresponding to about $N=7 \times 10^{5}$ proton spins, we estimate the ratio of the statistical to thermal polarizations to be approximately $P_{\text {stat }} / P_{\text {therm }} \simeq k_{B} T /\left(\mu B_{0} \sqrt{N}\right) \simeq 6$, where $k_{B}$ is the Boltzmann constant, $\mu$ is the proton magnetic moment, and $T=6 \mathrm{~K}$ is the spin temperature. In addition, unlike thermal measurements that require the ensemble to be repolarized between successive averages, the statistical polarization can be measured continuously.

The main disadvantage to detecting the statistical polarization is the random nature of the signal. Because the 
fluctuations have a zero mean value, the average signal cannot be used for NMR measurements. However, the fluctuations have a finite correlation time $\tau_{m}$, which depends on the NMR protocol used for spin detection. It is, therefore, possible to make use of the fluctuating spin polarization, provided that we encode and measure the spin ensemble faster than the statistical correlation time.

Spin measurements were performed using the modulated alternating gradients generated with currents (MAGGIC) protocol, which measures the $z$-axis component of the statistical spin polarization in the sample near the CFFGS [12]. In the MAGGIC protocol, an oscillating current through the CFFGS creates a magnetic field gradient $G=$ $d B_{z} / d x$ near the SiNW mechanical resonance frequency. The force produced by the spins in the presence of the field gradient induces Angstrom-scale motion of the SiNW, which is detected interferometrically.

In Appendix B, we provide a complete description of the MAGGIC protocol, along with the encoding scheme used for NMR measurements, the main result of which is encapsulated by Eq. (1). For a continuous spin distribution with density $\rho(\vec{r})$, the measured force correlation function is

$$
\begin{aligned}
C= & \frac{1}{2} \mu^{2} D^{2} e^{-\tau_{e} / T_{c}}\left(\frac{\tau_{m}}{\tau_{0}}\right)^{2}\left(1-e^{-\tau_{0} / \tau_{m}}\right)^{2} \\
& \times \int d \vec{r} \rho(\vec{r}) G^{2}(\vec{r}) \Theta(U(\vec{r})) \chi\left(\omega_{1}(\vec{r})\right),
\end{aligned}
$$

where $D$ and $\tau_{0}$ are the duty cycle and duration of the gradient modulation, and $\chi\left(\omega_{1}(\vec{r})\right)$ is an effective high-pass filter function, which sets a lower bound to the spin Rabi frequencies detected. The filtering procedure is discussed in Sec. IV of Ref. [13]. During the NMR encoding phase, which lasts a time $\tau_{e}$, a series of unitary transformations $U(\vec{r})$ are applied to a spin at position $\vec{r}$. The expectation value of the $z$-axis spin projection is given by the encoding function

$$
\Theta(U(\vec{r}))=\left\langle\uparrow\left|U^{\dagger}(\vec{r}) \sigma_{z} U(\vec{r})\right| \uparrow\right\rangle,
$$

where $\left\{\sigma_{x}, \sigma_{y}, \sigma_{z}\right\}$ are the Pauli matrices, and $T_{c}$ is the effective dephasing time over the encoding period.

\section{OPTIMAL CONTROL PULSE DESIGN}

In order to implement decoupling pulse sequences, one needs the ability to perform the same total spin rotation over the entire spin ensemble. In our system, this is not achievable with hard-pulse rotations because of the large field inhomogeneity produced by the CFFGS. Certain stateto-state transfers such as adiabatic passages can be achieved easily even in the presence of an inhomogeneous $B_{1}$ field [14], but these are insufficient for implementing decoupling sequences. OCT pulses have been used successfully in conventional NMR experiments to perform rotations despite various experimental constraints, including inhomogeneous $B_{1}$ fields.

The task of any OCT algorithm is to find a control sequence $a\left(t^{\prime}\right)$ over a period $0 \leq t^{\prime} \leq t$ that adheres to experimental constraints and generates the desired evolution for some ensemble of controlled systems. In the context of quantum control, $a\left(t^{\prime}\right)$ is generally an electromagnetic waveform, designed to generate a Hamiltonian evolution. Frequently, we are interested in a robust implementation of a particular unitary transformation $V$ for each member among the ensemble of interest; in this setting, we are dealing with an ensemble of nuclear spins. If $U_{i}\left[a\left(t^{\prime}\right)\right]$ is the unitary operation generated on the $i$ th spin at time $t$, we define a target function $\Phi\left(\left\{U_{i}\right\}\right)$, a functional of $a\left(t^{\prime}\right)$, that measures the total distance between $U_{i}\left[a\left(t^{\prime}\right)\right]$ and $V$ for the entire ensemble. The central problem of OCT is to find an $a\left(t^{\prime}\right)$ that minimizes $\Phi\left[a\left(t^{\prime}\right)\right]$. Many examples of numerical algorithms for solving this problem exist in the literature $[15,16]$.

One benefit of OCT algorithms over attempting to find $a\left(t^{\prime}\right)$ analytically stems from the fact that analytical solutions (e.g., composite hard pulses) are usually restricted to a single system rather than an ensemble. OCT pulses yield the same unitary operation $U_{i}\left[a\left(t^{\prime}\right)\right]=V$ at time $t$ for each member $i$ of the spin ensemble, even in the presence of markedly different individual spin Hamiltonians, by making use of the noncommutativity between rotations around different axes. The individual Bloch sphere trajectories for the spins experiencing $a\left(t^{\prime}\right)$ at different strengths because of $B_{1}$ variations can generally be very different-the ones experiencing greater $B_{1}$ values moving at higher rates and thereby completing a greater number of cycles around the Bloch sphere-yet a suitable $a\left(t^{\prime}\right)$ can make all these trajectories converge at some point $t$ in time. In Sec. VIII of Ref. [13], we illustrate this by giving a few examples of such convergent trajectories under a particular control sequence for several different Rabi strengths. For OCT algorithms, the presence of multiple systems merely adds extra steps into the computation, yet it typically does not affect their performance. Robust control in the presence of $B_{0}$ and $B_{1}$ inhomogeneities [17,18] and compensation for waveform distortions $[19,20]$ have both been demonstrated for conventional NMR measurements.

In this work, we used the gradient ascent pulse engineering algorithm [15], which prescribes an efficient way to find the functional derivative $\delta \Phi\left[a\left(t^{\prime}\right)\right] / \delta a\left(t^{\prime}\right)$ for piecewise constant control sequences $a\left(t^{\prime}\right)$. This converts the control search into a multivariate optimization problem. Given the functional derivative $\delta \Phi\left[a\left(t^{\prime}\right)\right] / \delta a\left(t^{\prime}\right)$ and a randomly generated initial guess $a_{0}(t)$, we used a simple multivariate gradient descent algorithm to reach the local minimum of $\Phi$ near $a_{0}(t)$. In practice, we generated a number of initial guesses until we found a waveform that yielded a suitably low $\Phi$. The details of this procedure are further discussed in Sec. V of Ref. [13]. In general, the length of the control sequence increases with the range of demands [e.g., range 
of targeted $B_{0}$ or $B_{1}$, bandwidth constraints for the waveform $a\left(t^{\prime}\right)$, etc.] required by the sequence. Additional demands typically require a larger number of initial guesses before a suitable sequence is found.

For this experiment, we searched for a pulse with a bandwidth of $10 \mathrm{MHz}$ that would perform a $\pi / 2$ rotation on all spins in a $\omega_{1}$ range, initially from $2 \pi \times 0.6 \mathrm{MHz}$ to $2 \pi \times 1.2 \mathrm{MHz}$, based on the experimental distribution of Rabi frequencies. First, we searched for a pulse that performed the desired unitary for this range of $\omega_{1}$, without explicitly averaging the dipolar Hamiltonian. The shortest pulse we found had a length of $t=7 \mu \mathrm{s}$, which is comparable to the measured $T_{2}^{*}=11 \mu \mathrm{s}$. To avoid dephasing over the duration of the pulse, we used a new method that incorporates AHT into the optimal control pulse design [21]. We added extra targets into our pulse optimization, by adding additional terms to $\Phi$, to minimize the leading-order terms of the homonuclear dipolar and chemical-shift Hamiltonians. Because of these added demands, the duration of the pulse increased from $7 \mu$ s to $13 \mu \mathrm{s}$; however, the dephasing during the pulse was dramatically reduced.
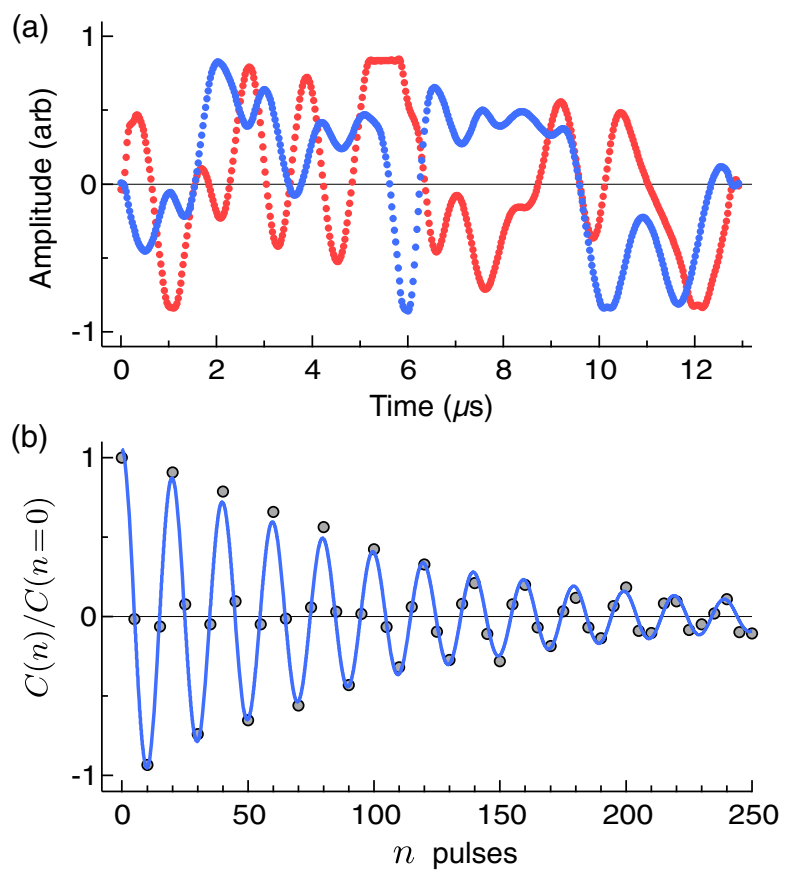

FIG. 2. Repeated $\Omega(\pi / 2)_{x}$ measurements. (a) Calculated $\Omega(\pi / 2)$ waveform. The two quadratures $a_{1}(t)$ (red line) and $a_{2}(t)$ (blue line) correspond to the modulation envelope of the $13-\mu$ s-long OCT-AHT pulse. The applied pulse is given by $a(t)=$ $a_{1}(t) \cos \left(\omega_{0} t+\alpha\right)+a_{2}(t) \sin \left(\omega_{0} t+\alpha\right)$, where $\omega_{0}$ is the Larmor frequency, and the phase $\alpha$ determines the direction of the effective field in the rotating frame. (b) Normalized spin correlation measurements. Here, $C(n)$ is the measured $z$-axis correlation [Eq. (1)] as a function of the number $n$ of sequential applications of the $\Omega(\pi / 2)_{x}$ pulse in the encoding sequence. The data are fit to the functional form $\cos (\pi n / 10) \exp (-c n)$. From the fit, we find $c=0.01$.
Details of the pulse optimization and calculated performance metrics are given in Secs. V and VII of Ref. [13].

To experimentally test the performance of the 13- $\mu$ s-long OCT-AHT pulse, we applied repeated $\Omega(\pi / 2)_{x}$ pulses to spins starting from the $z$ axis and measured the resulting $z$ axis magnetization. Here, $\Omega(\pi / 2)_{x}$ refers to an OCT-AHT pulse that performs a $\pi / 2$ rotation about the $x$ axis in the rotating frame. The data in Fig. 2(b) show the measured spin signal after sequential application of the $\Omega(\pi / 2)_{x}$ pulse, in increments of 5 pulses. The signal decays to $e^{-1}$ after 100 pulses, indicating that the operation works with $99 \%$ accuracy for spins starting from the $z$ axis.

This measurement serves as an initial demonstration of the power of OCT-AHT to produce high-fidelity unitary transformations tailored to our experimental conditions while averaging unwanted Hamiltonian terms to nearly zero. In this respect, these OCT-AHT pulses approximate ideal, infinitesimally short, hard pulses, making them perfectly suited to most NMR pulse sequences. It is worth noting that the solid-state NMR community has developed an immense range of pulse sequences for a wealth of experiments: imaging, chemical-shift spectroscopy, multidimensional (correlation) spectroscopy, and multinuclear experiments being just a few examples. Therefore, we expect the ability to implement any solid-state NMR pulse sequence, by substituting OCT-AHT pulses for hard rotation pulses, to have utility far beyond the experiments described in this paper. The next two sections demonstrate such an implementation of a particularly useful NMR sequence, the magic-echo sequence.

\section{MAGIC ECHOES}

To achieve high frequency resolution in NMR, it is necessary to decouple the spins of interest from the interactions that lead to dephasing. Many line-narrowing pulse sequences have been created by the NMR community to address a variety of different sources that cause decoherence. While the length and complexity of these sequences can vary greatly, their basic premise is the same. The sequence is designed to apply a series of unitary transformations on the spins, such that the average spin evolution by the unwanted Hamiltonians is minimized over the duration of the sequence. In the language of average Hamiltonian theory, the interaction Hamiltonian is rescaled in the toggling frame of the pulse sequence.

The magic-echo sequence is widely used by the solidstate NMR community to remove the dephasing caused by homonuclear dipole-dipole interactions. The ME sequence, shown schematically in Fig. 3, consists of two laboratoryframe evolution periods, each of length $\tau$, and a single rotating-frame evolution period of duration $4 \tau$. During the laboratory-frame evolution, the dipolar Hamiltonian has a scaling of +1 , while during the rotating-frame evolution, the scaling is $-1 / 2$. Thus, by making the length of the rotary 

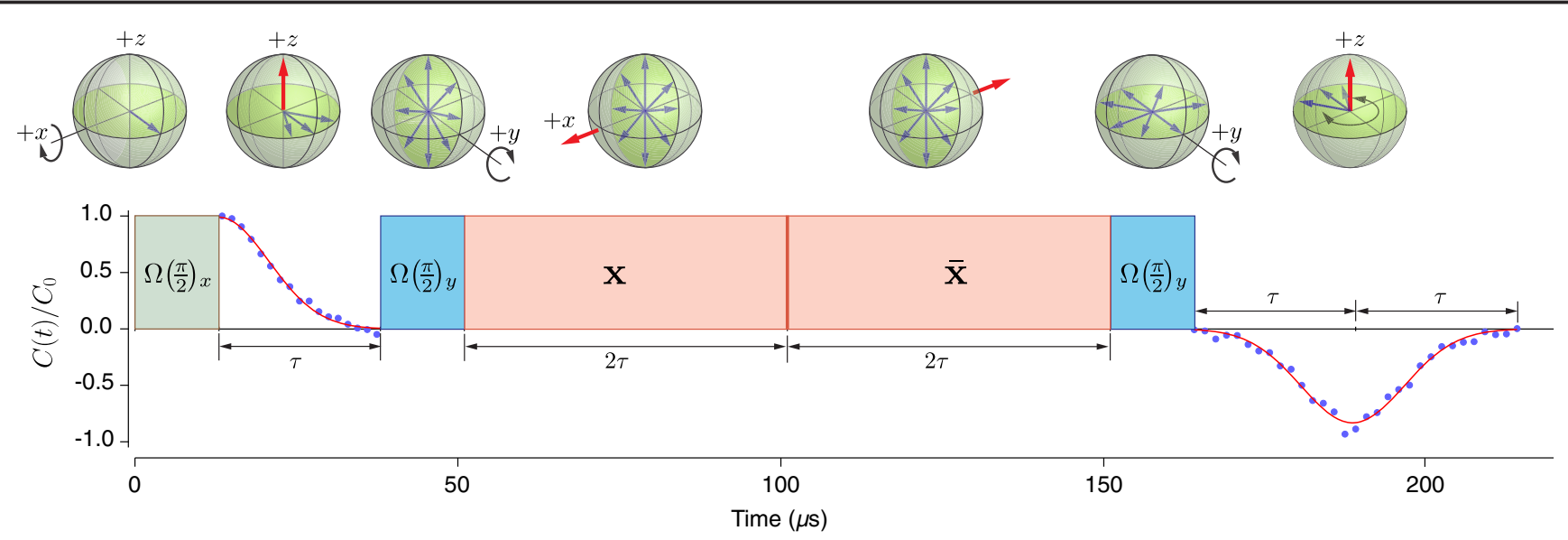

FIG. 3. Pulse diagram and data (blue circles) for the magic-echo pulse sequence performed using $\tau=25 \mu$ s and substituting $13-\mu \mathrm{s}-$ long OCT-AHT $\pi / 2$ pulses in place of hard-pulse rotations. The correlation measurements are performed after applying an $\Omega(\pi / 2)_{\bar{x}}$ pulse (not shown) at the times corresponding to the data points. The Bloch sphere diagrams above show the spin orientations (blue arrows) during the magic-echo sequence. The red arrows point in the direction of the external magnetic field during free evolution periods and in the direction of the effective field during the rotary echo.

evolution twice as long as the laboratory-frame evolution, the average dipole Hamiltonian is scaled to zero [22].

In our implementation of the ME sequence, the $\pi / 2$ spin rotations are accomplished using the $13-\mu$ s-long OCT-AHT pulses presented in Fig. 2(a). An $\Omega(\pi / 2)_{x}$ pulse creates the initial spin coherence. During the first laboratory-frame evolution period that follows, the proton spins dephase rapidly with a time constant $T_{2}^{*}=11 \mu \mathrm{s}$, primarily due to the strong dipole-dipole interactions in polystyrene. After the first evolution period $\tau$, an $\Omega(\pi / 2)_{y}$ is applied, followed by a rotary echo (RE) $(\mathbf{x}: \overline{\mathbf{x}})$, lasting a total time $4 \tau$. The RE consists of two equal-time, phase-alternated, Larmor frequency excitations applied in the $+x(\mathbf{x})$ and $-x(\overline{\mathbf{x}})$ directions, where the $x$ direction is referenced to the rotatingframe representation of the pulse. The equal-time phase alternation removes dephasing caused by the $B_{1}$ inhomogeneity. Following the RE, a second $\Omega(\pi / 2)_{y}$ pulse is applied. After the second laboratory-frame evolution period $\tau$, the echo is formed as the dipolar evolution is averaged to zero. In our implementation of the ME, two equal spin rotations $\Omega(\pi / 2)_{y}$ were used on either side of the RE, which also makes the average chemical-shift Hamiltonian zero over the duration of the pulse and causes an overall $\pi$ phase flip, resulting in the negative echo shown in Fig. 3. Removing the chemical-shift Hamiltonian has the added benefit of refocusing the dephasing caused by resonance offset effects.

To verify that the ME sequence modified using the OCTAHT rotations functions properly, we conduct a series of measurements to map out the spin coherence over the duration of the ME sequence. The encoding block consists of an initial $\Omega(\pi / 2)_{x}$ pulse, followed by the ME sequence. An $\Omega(\pi / 2)_{\bar{x}}$ pulse is applied at particular times within the ME sequence to rotate the $y$ component of the spins to the $z$ axis. In order to measure the envelope of the spin coherence, the time difference between the two $\Omega(\pi / 2)_{x, \bar{x}}$ pulses is chosen to be an integer multiple of the Larmor period. During the first laboratory-frame evolution period, we observe a decay envelope that is well described by a Gaussian: $C(t) / C_{0}=\exp \left(-\left(t / T_{2}^{*}\right)^{2}\right)$, with $T_{2}^{*}=11 \mu \mathrm{s}$, consistent with previous measurements in polystyrene [23]. Here, $C_{0}$ refers to the value of the correlation function at the start of the first free evolution period, and $t$ refers to the time difference between the end of the $\Omega(\pi / 2)_{x}$ pulse and the start of the $\Omega(\pi / 2)_{\bar{x}}$ pulse. After the RE, we observe the formation and subsequent decay of the magic echo. The data are fit to a Gaussian: $C(t) / C_{0}=-\exp \left(-\left[(t-\tau) / T_{2}^{*}\right]^{2}\right)$, with time $t$ referenced to the beginning of the second laboratory-frame evolution period. The observation of a strong echo signal confirms that the OCT-AHT pulses produce the expected spin rotation over the spin ensemble.

Symmetric magic-echo (SME) sequences are formed by combining several magic echoes around different axes. By doing so, the effect of certain pulse imperfections can be significantly reduced, resulting in longer coherence times than by simply repeating a single magic echo. SME sequences have been demonstrated to have excellent linenarrowing capabilities [24] and can be used for chemicalshift spectroscopy and Fourier-transform imaging. We test the SME4 and SME16 sequences, both of which remove chemical-shift effects, by applying the sequences repeatedly and observing the decay of the spin coherence.

The encoding sequence consists of an initial $\Omega(\pi / 2)_{x}$ pulse to create the spin coherence, followed by the SME sequence, and a final $\Omega(\pi / 2)_{\bar{x}}$ pulse to rotate the spin magnetization from the $y$ axis back to the $z$ axis. Figure 4 shows the normalized spin echo envelope obtained by repeated application of the SME4 and SME16 pulse sequences, block diagrams of the pulse sequences, and a table of calculated NMR linewidths in units of Hz. The spin coherence time was determined by fitting the envelope of 

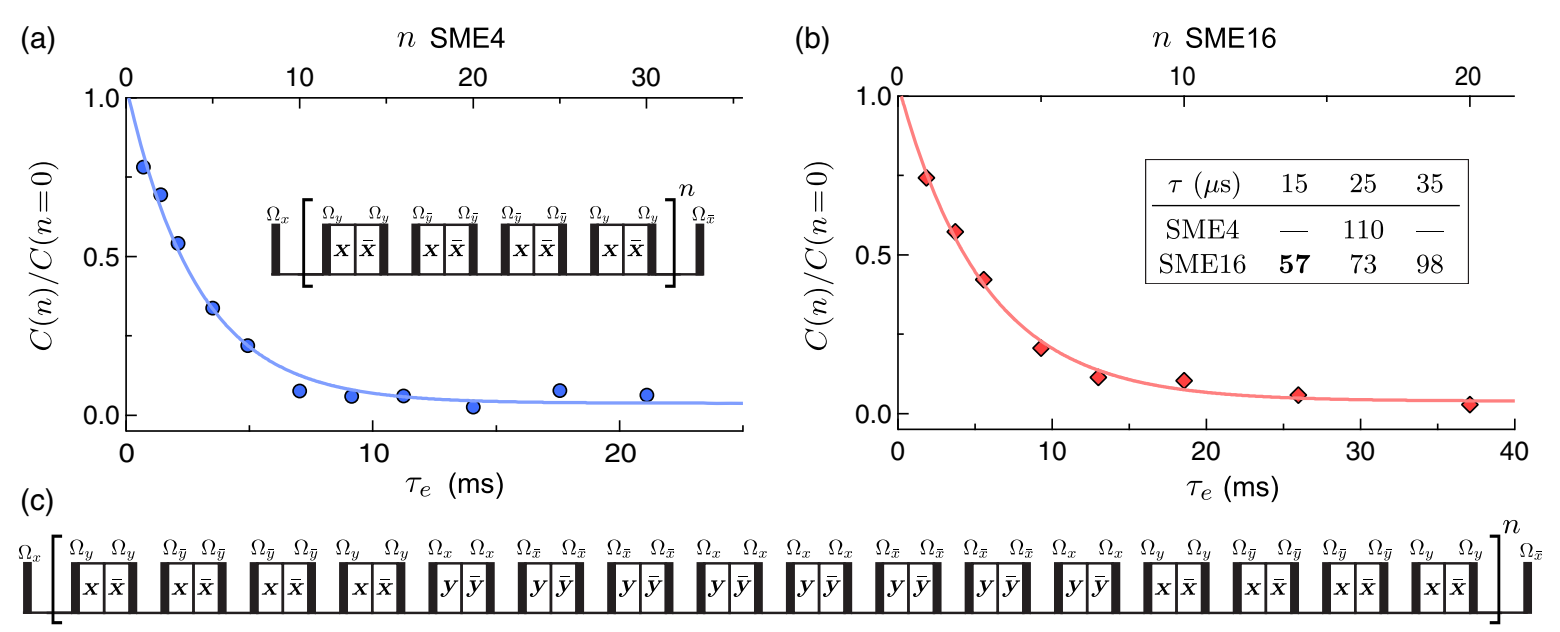

FIG. 4. Normalized spin echo measurements as a function of total encoding time (bottom axis) and number of sequences repeated (top axis) for sequential applications of an SME4 decoupling sequence. The time axis represents the total encoding time, which includes the free evolution and rotary evolution periods, and the duration of the OCT-AHT pulses. Exponential decay curves are fit to the data and used to determine the coherence time $T_{c}$. (a) SME4 data $\left(\tau=25 \mu \mathrm{s}, T_{c}=2.9 \mathrm{~ms}\right)$. (Inset) SME4 pulse-sequence block diagram. (b) SME16 data ( $\tau=15 \mu \mathrm{s}, T_{c}=5.6 \mathrm{~ms}$ ). (Inset) Table of calculated linewidths $\Delta \nu$ in units of Hz for sequential application of different SME sequences. (c) SME16 pulse-sequence block diagram.

the SME data to an exponential decay. An exponential decay of the coherence corresponds to a Lorentzian frequency distribution whose linewidth is given by $\Delta \nu=1 /\left(\pi T_{c}\right)$, where $T_{c}$ is the spin coherence time, equal to the time constant of the exponential decay. We observe that the SME16 sequence performs slightly better than the SME4, with the longest spin coherence time $T_{c}=5.6 \mathrm{~ms}$ (corresponding to a linewidth of $\Delta \nu=57 \mathrm{~Hz}$ ) observed for SME16 $(\tau=15 \mu \mathrm{s})$. For all measurements, the value of $\tau$ was chosen to be longer than $T_{2}^{*}$ to avoid spin-lock effects $[25,26]$.

\section{IMAGING}

In previous work, we demonstrated that the static and $\mathrm{rf}$ field gradients generated by the CFFGS could be used to encode spins in two dimensions [3]. Our goal in this section is to demonstrate the ability to combine the long spin coherence times achieved by the SME sequence with the intense field gradients produced by the CFFGS to demonstrate state-of-the-art imaging resolution of nuclear spins. The long spin evolution periods within the ME sequence are ideal for imaging [27]. The $B_{0}$ gradient pulses applied during the laboratory frame evolution periods and $B_{1}$ gradient pulses applied during the RE can be used to phase-encode spin positions along contours of constant Larmor and Rabi frequencies, respectively.

Here, we use the $B_{1}$ gradient generated by the CFFGS to encode spin locations in one dimension. For this work, we use an SME4 ( $\tau=15 \mu \mathrm{s})$ sequence with the RE portion modified for $B_{1}$ encoding. The RE modification, shown in Fig. 5(a), consists of applying the ( $\mathbf{x})$ rf field for more or less time than the $(\overline{\mathbf{x}})$ rf field while maintaining a total rotary evolution period of $4 \tau$ in each ME sequence. With the modified RE, the spins evolve in the $B_{1}$ gradient for a time $t=8 \Delta T$ within a single SME4 sequence, where $\Delta T$ is the offset introduced to each RE [Fig. 5(a)]. In the rotatingframe representation, the modified SME4 sequence applies the unitary $U(\vec{r}, t)=\exp \left(-i \sigma_{x} \theta(\vec{r}, t) / 2\right)$, which rotates the spins at position $\vec{r}$ by an angle $\theta(\vec{r}, t)=\gamma_{p} B_{1}(\vec{r}) t=\omega_{1} t$ with respect to the $x$ axis. The corresponding encoding function is given by $\Theta(U(\vec{r}, t))=\cos (\theta(\vec{r}, t))$.

Prior to the imaging measurements, the tip was repositioned over the CFFGS, which increases the maximum Rabi frequency from 1.2 MHz to $1.6 \mathrm{MHz}$ for spins closest to the CFFGS. For the imaging experiments, we designed a new 7.5- $\mu$ s-long OCT-AHT pulse to target spins within the Rabi frequency range of $0.9 \mathrm{MHz}$ to $1.75 \mathrm{MHz}$. These new pulses were used in the SME4 sequence to collect the imaging data shown in Fig. 5.

To determine the spin distribution as a function of Rabi frequency, we incremented $\Delta T$ and measured the corresponding spin correlation data $C(t)$. Figure 5(b) shows three different measurements. For the data points shown using the red diamonds and blue circles, 21 points were measured with a maximum evolution time of $t_{\max }=6.7 \mu \mathrm{s}$, corresponding to a Nyquist frequency of $1.5 \mathrm{MHz}$, and a frequency resolution of $\Delta f=75 \mathrm{kHz}$. For the data shown using the green triangles, 31 points were measured with a maximum evolution time of $t_{\max }=20 \mu \mathrm{s}$, corresponding to a Nyquist frequency of $750 \mathrm{kHz}$, and $\Delta f=25 \mathrm{kHz}$.

Data were acquired using a time-proportional phase increment procedure, which shifted the frequency range to include spins experiencing the highest Rabi frequencies $(\sim 1.6 \mathrm{MHz})$. To shift the frequency range by $\Delta \omega$, the modified SME4 sequences were applied with the relative phase between the beginning and ending $\Omega_{x}$ and $\Omega_{\bar{x}}$ pulses 


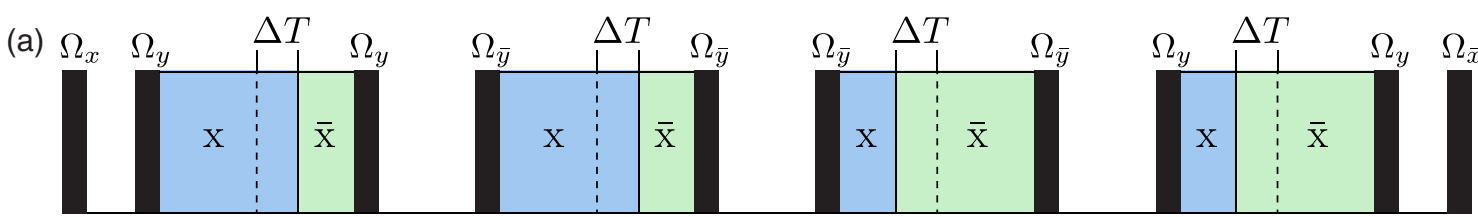

(b)

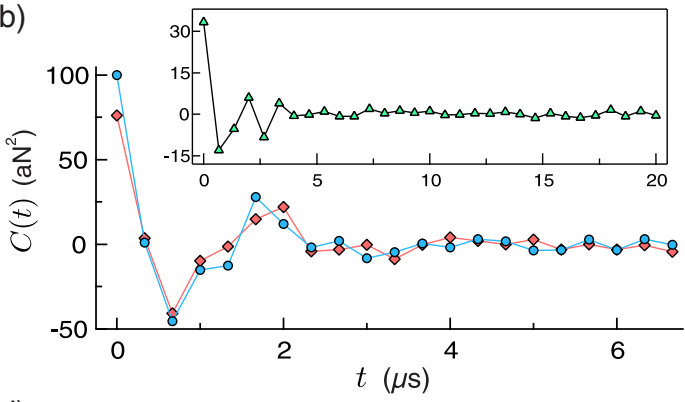

(c)

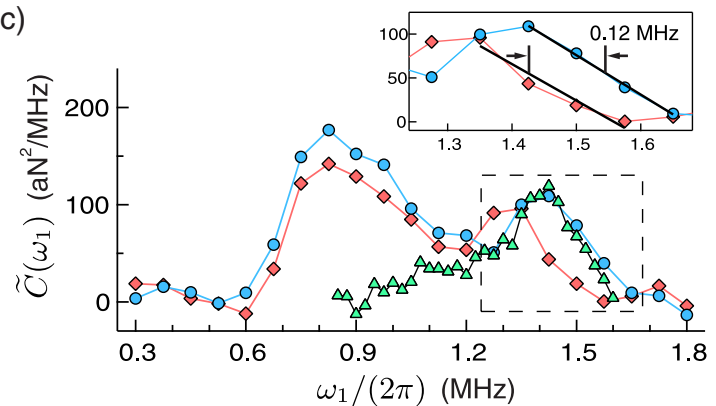

(d)

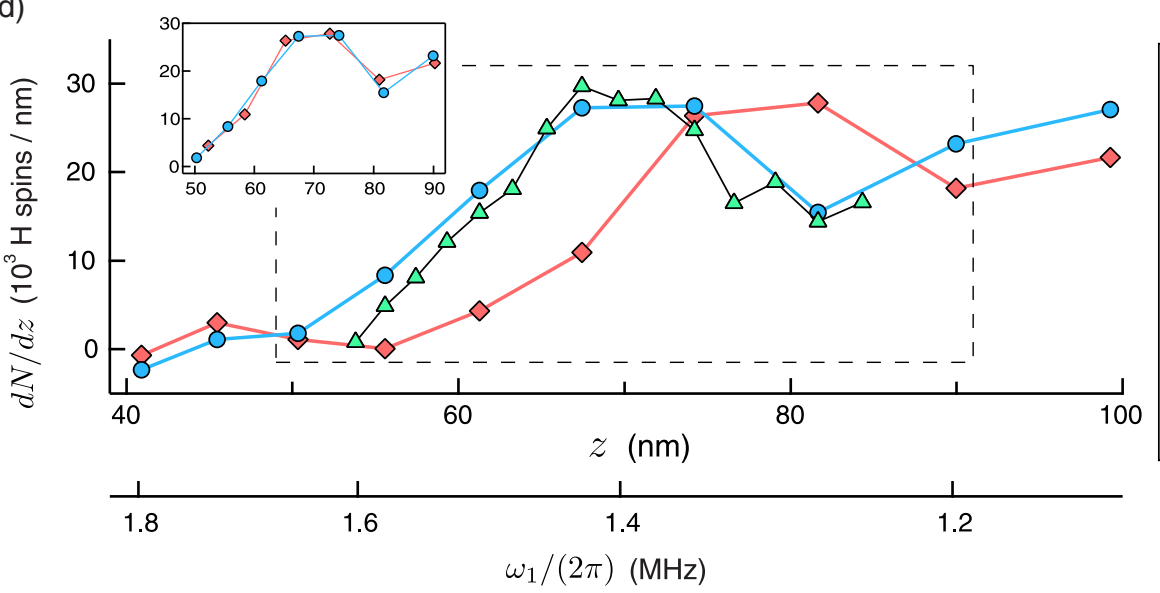

\begin{tabular}{|l}
$\Delta t_{\max }=20 \mu \mathrm{s}$ \\
$\Delta f=25 \mathrm{kHz}$ \\
\\
Tip-surface distance: $55 \mathrm{~nm}$ \\
$t_{\max }=6.7 \mu \mathrm{s}$ \\
$\Delta f=75 \mathrm{kHz}$ \\
\\
\\
Tip-surface distance: $65 \mathrm{~nm}$ \\
$t_{\text {max }}=6.7 \mu \mathrm{s}$ \\
$\Delta f=75 \mathrm{kHz}$
\end{tabular}

FIG. 5. One-dimensional imaging. (a) Modified SME4 spin sequence used for one-dimensional imaging includes time offsets ( $\Delta T$ ) during rotary evolution periods to encode Rabi frequency information. (b) Spin correlation data $C(t)$ after a single modified SME4 sequence as a function of rotary evolution time $t=8 \Delta T$. The data displayed using the blue and red points were acquired with $0.33-\mu \mathrm{s}$ steps at a tip-surface separation of $55 \mathrm{~nm}$ and $65 \mathrm{~nm}$, respectively. The frequency resolution for both data sets is $75 \mathrm{kHz}$. (Inset) The green data points were acquired with $0.66-\mu$ s steps at a tip-surface separation of $55 \mathrm{~nm}$. The frequency resolution for these data is $25 \mathrm{kHz}$. (c) Spectral density data. The sharp decrease in signal observed below $0.7 \mathrm{MHz}$ for the $75-\mathrm{kHz}$ resolution data and $1.2 \mathrm{MHz}$ for the $25-\mathrm{kHz}$ resolution data is due to the high-pass filter discussed in Sec. IV of Ref. [13]. (d) One-dimensional proton density as a function of height above the CFFGS. Tip-surface separation and frequency step size are the same for each data set as those in part (c). (Inset) $75-\mathrm{kHz}$ resolution data, with the $65-\mathrm{nm}$ data offset by $9 \mathrm{~nm}$ in the negative $z$ direction.

[Fig. 5(a)] offset by an amount proportional to the total evolution time: $\Delta \alpha=\Delta \omega t$ [see Fig. 2(a) for the definition of the angle $\alpha$ ]. For the $t_{\max }=6.7 \mu \mathrm{s}$ and $t_{\max }=20 \mu \mathrm{s}$ data, the frequency shift was chosen to be $\Delta \omega /(2 \pi)=300 \mathrm{kHz}$ and $\Delta \omega /(2 \pi)=850 \mathrm{kHz}$, respectively. The corresponding frequency range was $0.3-1.8 \mathrm{MHz}\left(t_{\max }=6.7 \mu \mathrm{s}\right.$ data $)$ and $0.85-1.6 \mathrm{MHz}\left(t_{\max }=20 \mu\right.$ s data.) To prevent aliasing of the spin signal from those spins whose Rabi frequencies were below the minimum frequency, we implemented a high-pass filter by modifying the AFP pulses used in the MAGGIC readout. In Sec. IV of Ref. [13], we discuss the filtering procedure and compare the calculated filter response with the measured signal spectral densities.

The force signal spectral density as a function of Rabi frequency was calculated by performing the discrete cosine transform of the time-domain data shown in Fig. 5(b). Figure 5(c) shows the discrete cosine transform $\tilde{C}\left(\omega_{1}\right)$, normalized to represent spectral density. To construct the one-dimensional spin density $d N / d z$ shown in Fig. 5(d), we first converted the frequency-domain data plotted in Fig. 5(c) to be a function of position. In general, the Rabi frequency varies in all three directions. However, near the center of the constriction, the variation is mostly in the $z$ direction. The simulated field distributions (Fig. S3) indicate that the magnitude of $B_{1}$ varies by less than $2 \%$ in a $50 \times 50(\mathrm{~nm})^{2}$ region in the $x y$ plane centered on the constriction. To calculate the function $\omega_{1}(z)$, we used the simulated magnetic field data shown in Fig. 1(c) and took the value of $\omega_{1}$ at each vertical position to be the average over a $50 \times 50(\mathrm{~nm})^{2}$ region in the $x$ and $y$ dimensions. We 
then used Eq. (1) to convert the force signal into an equivalent number of spins using the simulated readout gradient $d B_{z}(z) / d x$. The function $d B_{z}(z) / d x$ was calculated in an identical fashion to the function $\omega_{1}(z)$. Spin density was calculated by dividing the number of spins by the slice thickness.

To validate the calculated field values, we repeated the imaging measurement at two different vertical locations of the tip by translating the sample by $10 \mathrm{~nm}$ using a calibrated piezoelectric actuator. Figure 5(c) shows the force signal spectral density as a function of Rabi frequency obtained for a tip-surface separation of $55 \mathrm{~nm}$ (blue curve) and $65 \mathrm{~nm}$ (red curve). We observe that the frequency distribution for the 65$\mathrm{nm}$ separation data is shifted to lower Rabi frequencies relative to the 55-nm separation data, with the shift being largest for spins experiencing the highest $B_{1}$ fields (those closest to the CFFGS). The inset above Fig. 5(c) shows an average frequency offset of $\Delta f=0.12 \mathrm{MHz}$ for the frequency range 1.4-1.6 MHz. Using the frequency shift and the applied tip offset of $\Delta z=10 \mathrm{~nm}$, we estimate the average $B_{1}$ gradient in this frequency range to be $d B_{1} / d z \simeq 2 \pi \Delta f /\left(\gamma_{p} \Delta z\right)=2.8 \mathrm{G} / \mathrm{nm}$, which is in excellent agreement with the calculated value of $d B_{1} / d z=2.9 \mathrm{G} / \mathrm{nm}$ using the COMSOL simulation over the same frequency range.

As a complementary validation of the simulated field distribution, we numerically adjusted the relative offset between the two calculated spin-density curves and varied the offset to minimize the mean-square distance between the two data sets. Using this approach, we estimate that offset to be $9 \pm 2 \mathrm{~nm}$, in good agreement with the applied offset. The inset in Fig. 5(d) shows the 55-nm data along with the 65-nm data offset by $9 \mathrm{~nm}$ in the negative $z$ direction.

From the data shown in Fig. 5(b), we find that the amplitude of the correlation signal decreases substantially for $B_{1}$ gradient evolution times longer than about $4 \mu \mathrm{s}$. To verify that the reduction in $C(t)$ was not caused by a degradation in the refocusing properties of the modified SME4 sequence, we applied two back-to-back modified SME4 pulses, changing the sign of $\Delta T$ in the two pulses to undo the evolution in the $B_{1}$ gradient. We chose $\Delta T=3.12 \mu \mathrm{s}$; thus, the spins evolved in the $B_{1}$ gradient for a total time of $25 \mu$ s within a single modified SME4 sequence. We compared the spin signal after applying a pair of unmodified SME4 sequences $(\Delta T=0)$ with the signal after applying the pair of modified SME4 sequences and found no change in the magnitude of the signal, confirming that the modified SME4 pulses retained the refocusing properties of the original SME4 sequence. Importantly, the test also confirmed that the spin coherence is maintained during the $50 \mu$ s period that the spins evolved in the $B_{1}$ gradient of the two SME4 pulses.

To take advantage of the enhanced coherence time, we evolved the spins up to $t_{\max }=20 \mu \mathrm{s}$ in the $B_{1}$ gradient. For the high-resolution data, 31 points were measured with an averaging time of 3 hours per point [Fig. 5(b) inset]. The average noise for the discrete cosine transform of these data was $0.20 \mathrm{aN}^{2}$ in a $25-\mathrm{kHz}$ bandwidth. For the spins experiencing the highest readout gradients of $1 \times 10^{6} \mathrm{~T} / \mathrm{m}$ near the tip, this force noise corresponds to a statistical polarization of approximately $\sqrt{N}=55$ proton spins.

Although $t_{\max }$ was approximately twice as long as $T_{2}^{*}$, it was still substantially shorter than the maximum rotary evolution time $(16 \tau=240 \mu \mathrm{s})$ available using a single modified SME4 sequence. Had we evolved the spins up to $240 \mu$ s using an average encoding gradient of $2.8 \mathrm{G} / \mathrm{nm}$, the average slice thickness in a 30-nm region of the tip would have been reduced from approximately $2 \mathrm{~nm}$ to less than $2 \AA$. However, imaging this particular sample with 2 - $\AA$ resolution with the same signal-to-noise ratio would have made the averaging times impractical. Furthermore, because the sample contained little spin-density variation on length scales below about $10 \mathrm{~nm}$, imaging with subnanometer slice thickness would not have significantly improved the image resolution. In the next section, we discuss methods to significantly enhance detection sensitivity, which would reduce averaging times, enabling full advantage to be taken of the long coherence times demonstrated in this work.

\section{CONCLUSION}

With this work, we have successfully brought together the use of intense magnetic fields and magnetic field gradients generated by the CFFGS, necessary for both efficient detection and imaging of nanoscale spin ensembles, and OCT-AHT pulses that yield high-fidelity control in a seemingly very challenging control setting. Importantly, with the example of magic-echo sequences, we have demonstrated an ability to adapt existing solid-state NMR pulse sequences to our nanoscale experiments by simply substituting their respective hard pulses with OCT-AHT pulses. The line-narrowing ability we demonstrated for a densely packed proton sample by the use of SME sequences was comparable to some of the best conventional NMR experiments [24]. We believe that the capability to use existing solid-state NMR protocols along with high-sensitivity spin detection is very powerful and will enable a variety of new experiments on nanoscale spin ensembles beyond the examples presented here, of which atomic-scale NMR diffraction [28] and multidimensional (correlation) spectroscopy could prove to be particularly transformative.

The very high field gradients and long coherence times we demonstrated provided us with a technical ability to encode spatial information on the Ångstrom scale, while the resolution for a previous imaging experiment with a similar experimental setup [3] had been limited to $10 \mathrm{~nm}$ because of short coherence times. Conversely, the onedimensional slice thickness for the imaging experiment was chosen to be approximately $2 \mathrm{~nm}$, in part because of limitations in spin-detection sensitivity and the lack of 
sample features on shorter length scales. One way to greatly improve the imaging resolution for a given measurement period is to utilize the fact that electron spin polarization in our field $(1.13 \mathrm{~T})$ and temperature $(6 \mathrm{~K})$ is $12 \%$. Hence, a perfect transfer of spin polarization from the electrons to the nuclei using one of the many dynamical nuclear polarization schemes would enhance our signal levels by a factor of nearly 100. By going to lower temperatures, polarization may be further enhanced. Furthermore, the techniques presented here can be readily extended to threedimensional imaging by fabricating additional modulation coils near the CFFGS for applying gradients in three directions.

\section{ACKNOWLEDGMENTS}

This work was undertaken thanks in part to funding from the U.S. Army Research Office through Grant No. W911NF1610199, and from the Canada First Research Excellence Fund (CFREF). Additional support was provided by the Department of Physics at the University of Illinois. Research was carried out in part in the Frederick Seitz Materials Research Laboratory Central Research Facilities at the University of Illinois. D. G. C. acknowledges the Canada Excellence Research Chairs Program. R. B. and D. G. C. acknowledge support from the Natural Sciences and Engineering Research Council of Canada (NSERC), the Canadian Institute for Advanced Research, the province of Ontario, and Industry Canada. L. L. and N. J. acknowledge support from NSF Grants No. DMR-1611341 and No. DMR-1308654. R. B. would like to thank Ben Yager for performing ESR measurements of paramagnetic defects in silicon nanowires.

W. R. and H. H. contributed equally to this work.

\section{APPENDIX A: SILICON NANOWIRE RESONATORS}

The SiNWs were modified using a procedure developed to split the frequencies of the two lowest-frequency flexural modes while maintaining high-quality factors. The details related to mode engineering of SiNW resonators are discussed in Sec. I of Ref. [13]. The modified SiNW resonator used for this experiment was approximately $20 \mu \mathrm{m}$ long, with tip dimensions of $60 \mathrm{~nm} \times 80 \mathrm{~nm}$. At $4.2 \mathrm{~K}$, the two fundamental flexural modes had resonance frequencies $f_{1,2}=(315 \mathrm{kHz}, 369 \mathrm{kHz})$, quality factors $Q_{1,2}=(8000,8300)$, and spring constant $k \approx 1.0 \times 10^{-4} \mathrm{~N} / \mathrm{m}$. We used a $2-\mu \mathrm{m}$-wavelength laser interferometer polarized vertically along the length of the SiNW to measure its displacement [29]. The laser was focused to a roughly $3-\mu \mathrm{m}$-diameter spot near the center of the SiNW. The lower-frequency mode $\left(f_{1}\right)$ was used for spin measurements because its oscillation direction was better aligned with the interferometer.

\section{APPENDIX B: MAGGIC SPIN MEASUREMENT PROTOCOL}

Here, we present a discussion of the MAGGIC spindetection protocol and a derivation of Eq. (1). NMR measurements are performed using the sequence shown in Fig. 6(a). In the first part of the sequence, the MAGGIC waveform is applied for a duration $\tau_{0}=80 \mathrm{~ms}$, with $\tau_{0}$ chosen to be much shorter than the experimentally determined average correlation time $\tau_{m}=0.6 \mathrm{~s}$ of the statistical fluctuations using the MAGGIC protocol. The MAGGIC waveform [Fig. 6(b)] consists of two periods, during which the gradient $d B_{z} / d x$ is modulated at the resonance frequency of the cantilever (light blue waveform). A $\pi$ phase shift is introduced between the two modulation periods to ensure that the MAGGIC waveform does not contain Fourier components at the cantilever resonance frequency, thus eliminating any spurious electrical coupling to the cantilever. An adiabatic full passage (AFP) pulse (red waveform), applied between the two gradient modulation periods, inverts the $z$-axis component of the spins. By inverting both the spin orientation and the phase of the gradient modulation, each spin in the measurement ensemble produces a peak force $F_{0}=\mu D G / \sqrt{2}$ at the resonance frequency of the cantilever, where $D$ is the duty cycle of the

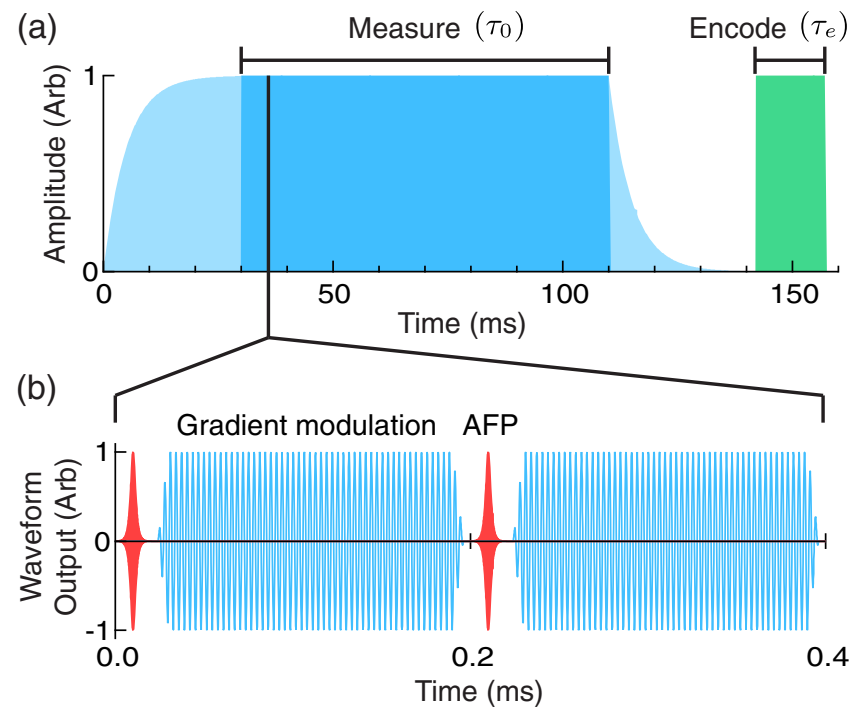

FIG. 6. NMR encoding and spin detection. (a) Timing diagram for a single MAGGIC measurement sequence. To minimize spurious mechanical excitation of the SiNW, the gradient modulation is turned on and off with an exponential envelope having a time constant of $Q_{1} /\left(\pi f_{1}\right)=8 \mathrm{~ms}$ (light blue regions.) The quality factor $Q_{1}$ and resonance frequency $f_{1}$ of the SiNW resonator are discussed in Appendix A. Dark blue areas indicate the readout period, during which the AFPs and the gradient modulation were applied simultaneously. The encoding time $\tau_{e}$ varied between a few microseconds up to several milliseconds, depending on the desired encoding sequence. (b) Diagram showing the MAGGIC waveform. 
gradient modulation and $G=d B_{z} / d x$ is the peak magnetic field gradient at the location of the spin.

Using the two force measurements before and after a given encoding period, we construct the force correlation function for a single spin at position $\vec{r}$,

$$
\begin{aligned}
c(U(\vec{r}))= & \frac{1}{2} \mu^{2} D^{2} G^{2}(\vec{r}) \Theta(U(\vec{r})) \chi\left(\omega_{1}(\vec{r})\right) e^{-\tau_{e} / T_{c}} \\
& \times \frac{1}{\tau_{0}^{2}}\left\langle\int_{0}^{\tau_{0}} h\left(\vec{r}, t^{\prime}\right) d t^{\prime} \int_{0}^{\tau_{0}} h\left(\vec{r}, t+\tau_{0}\right) d t\right\rangle,
\end{aligned}
$$

where $\langle\ldots\rangle$ refers to the ensemble average of measurements. In Eq. (B1), we model the instantaneous spin projections onto the $z$ axis as a stationary zero-mean Markovian process described by the random telegraph function $h(\vec{r}, t)$. Here, $h(\vec{r}, t)$ is a stochastic function that, at any instant, takes values \pm 1 . For some initial value $h(\vec{r}, 0)= \pm 1$, the probability of $N$ sign flips over an interval $[0, t]$ is given by the Poisson process, such that $\operatorname{prob}(N)=\left(t / \tau_{m}\right)^{N} \exp \left(-t / \tau_{m}\right) / N ! ; \quad$ this property determines the correlation function $\left\langle h\left(\vec{r}, t^{\prime}\right) h(\vec{r}, t)\right\rangle=$ $\exp \left(-\left|t-t^{\prime}\right| \tau_{m}\right)$. The high-pass filter function $\chi\left(\omega_{1}(\vec{r})\right)$ arises because of imperfections in implementing the AFPs; the passage has the desired effect of performing a spin flip only if the adiabatic condition is fulfilled, which in turn requires the spin Rabi frequency to be sufficiently high. The AFP amplitude and modulation are chosen such that $\chi\left(\omega_{1}(\vec{r})\right) \approx 1$ for the spins of interest. However, for spins with low enough $\omega_{1}(\vec{r})$, we have $\chi\left(\omega_{1}(\vec{r})\right) \approx 0$. A detailed discussion of the filtering property and a derivation of $\chi\left(\omega_{1}(\vec{r})\right)$ is presented in Sec. IV of Ref. [13].

In writing Eq. (B1), we have assumed that the encoding period $\tau_{e}$ is much shorter than the spin-lattice relaxation time $T_{1}$ and that the statistical correlations decay only during the time that the MAGGIC detection is made [dark blue areas in Fig. 6(a)]. Equation (1) of the main section is a generalization of Eq. (B1) for a continuous spin distribution. In making this generalization, we assume that the spin fluctuations from different parts of the sample are, on average, uncorrelated.

[1] D. Rugar, R. Budakian, H. J. Mamin, and B. W. Chui, Single Spin Detection by Magnetic Resonance Force Microscopy, Nature (London) 430, 329 (2004).

[2] C. L. Degen, M. Poggio, H. J. Mamin, C. T. Rettner, and D. Rugar, Nanoscale Magnetic Resonance Imaging, Proc. Natl. Acad. Sci. U.S.A. 106, 1313 (2009).

[3] J. M. Nichol, T. R. Naibert, E. R. Hemesath, L. J. Lauhon, and R. Budakian, Nanoscale Fourier-Transform Magnetic Resonance Imaging, Phys. Rev. X 3, 031016 (2013).

[4] D. Rugar, H. J. Mamin, M. H. Sherwood, M. Kim, C. T. Rettner, K. Ohno, and D. D. Awschalom, Proton Magnetic
Resonance Imaging Using a Nitrogen-Vacancy Spin Sensor, Nat. Nanotechnol. 10, 120 (2015).

[5] H. J. Mamin, M. Kim, M. H. Sherwood, C. T. Rettner, K. Ohno, D. D. Awschalom, and D. Rugar, Nanoscale Nuclear Magnetic Resonance with a Nitrogen-Vacancy Spin Sensor, Science 339, 557 (2013).

[6] T. Staudacher, F. Shi, S. Pezzagna, J. Meijer, J. Du, C. A. Meriles, F. Reinhard, and J. Wrachtrup, Nuclear Magnetic Resonance Spectroscopy on a (5-nanometer) ${ }^{3}$ Sample Volume, Science 339, 561 (2013).

[7] A. O. Sushkov, I. Lovchinsky, N. Chisholm, R. L. Walsworth, H. Park, and M. D. Lukin, Magnetic Resonance Detection of Individual Proton Spins Using Quantum Reporters, Phys. Rev. Lett. 113, 197601 (2014).

[8] C. L. Degen, Q. Lin, A. Hunkeler, U. Meier, M. Tomaselli, and B. H. Meier, Microscale Localized Spectroscopy with a Magnetic Resonance Force Microscope, Phys. Rev. Lett. 94, 207601 (2005).

[9] C. L. Degen, Q. Lin, and B. H. Meier, Dipolar Spin Echoes in Magnetic Resonance Force Microscopy, Phys. Rev. B 74, 104414 (2006).

[10] R. Verhagen, A. Wittlin, C. W. Hilbers, H. van Kempen, and A.P. M. Kentgens, Spatially Resolved Spectroscopy and Structurally Encoded Imaging by Magnetic Resonance Force Microscopy of Quadrupolar Spin Systems, J. Am. Chem. Soc. 124, 1588 (2002).

[11] K. W. Eberhardt, C. L. Degen, A. Hunkeler, and B. H. Meier, One- and Two-Dimensional NMR Spectroscopy with a Magnetic-Resonance Force Microscope, Angew. Chem. 47, 8961 (2008).

[12] J. M. Nichol, E. R. Hemesath, L. J. Lauhon, and R. Budakian, Nanomechanical Detection of Nuclear Magnetic Resonance Using a Silicon Nanowire Oscillator, Phys. Rev. B 85, 054414 (2012).

[13] See Supplemental Material at http://link.aps.org/ supplemental/10.1103/PhysRevX.8.011030 for additional experimental details and calculations.

[14] M. Garwood and L. DelaBarre, The Return of the Frequency Sweep: Designing Adiabatic Pulses for Contemporary NMR, J. Magn. Reson. 153, 155 (2001).

[15] N. Khaneja, T. Reiss, C. Kehlet, T. Schulte-Herbrüggen, and S. J. Glaser, Optimal Control of Coupled Spin Dynamics: Design of NMR Pulse Sequences by Gradient Ascent Algorithms, J. Magn. Reson. 172, 296 (2005).

[16] P. Doria, T. Calarco, and S. Montangero, Optimal Control Technique for Many-Body Quantum Dynamics, Phys. Rev. Lett. 106, 190501 (2011).

[17] T. W. Borneman, M. D. Hürlimann, and D. G. Cory, Application of Optimal Control to CPMG Refocusing Pulse Design, J. Magn. Reson. 207, 220 (2010).

[18] J.-S. Li, J. Ruths, T.-Y. Yu, H. Arthanari, and G. Wagner, Optimal Pulse Design in Quantum Control: A Unified Computational Method, Proc. Natl. Acad. Sci. U.S.A. 108, 1879 (2011).

[19] T. W. Borneman and D. G. Cory, Bandwidth-Limited Control and Ringdown Suppression in High-Q Resonators, J. Magn. Reson. 225, 120 (2012).

[20] I. N. Hincks, C. E. Granade, T. W. Borneman, and D. G. Cory, Controlling Quantum Devices with Nonlinear Hardware, Phys. Rev. Applied 4, 024012 (2015). 
[21] H. Haas, D. Puzzuoli, and D. Cory (unpublished).

[22] W.-K. Rhim, A. Pines, and J.S. Waugh, Time-Reversal Experiments in Dipolar-Coupled Spin Systems, Phys. Rev. B 3, 684 (1971).

[23] M. F. Froix, D. J. Williams, and A. O. Goedde, NMR Relaxation Time Studies of Polystyrene, Macromolecules 9, 354 (1976).

[24] G. S. Boutis, P. Cappellaro, H. Cho, C. Ramanathan, and D. G. Cory, Pulse Error Compensating Symmetric MagicEcho Trains, J. Magn. Reson. 161, 132 (2003).

[25] S. Matsui, Suppressing the Zero-Frequency Artifact in Magic-Sandwich-Echo Proton Images of Solids, J. Magn. Reson. 98, 618 (1992).
[26] S. Matsui and H. Miura, 1H-13C Cross-Polarization Using a Modified Magic Echo Sequence for 1H Spin Locking, Chem. Phys. Lett. 242, 163 (1995).

[27] S. Hafner, D. E. Demco, and R. Kimmich, Magic Echoes and NMR Imaging of Solids, Solid State Nucl. Magn. Reson. 6, 275 (1996).

[28] P. Mansfield and P. K. Grannell, NMR "Diffraction" in Solids?, J. Phys. C 6, L422 (1973).

[29] J. M. Nichol, E. R. Hemesath, L. J. Lauhon, and R. Budakian, Displacement Detection of Silicon Nanowires by Polarization-Enhanced Fiber-Optic Interferometry, Appl. Phys. Lett. 93, 193110 (2008). 